\title{
BMAL1 may be involved in angiogenesis and peritumoral cerebral edema of human glioma by regulating VEGF and ANG2
}

\author{
Fan Wang ${ }^{1, *}$, CaiYan $\mathrm{Li}^{2,}{ }^{*}$, Fei Han ${ }^{3}$, LvAn Chen ${ }^{1}$, Ling $\mathrm{Zhu}^{1}$ \\ ${ }^{1}$ Department of Neurosurgery, The Affiliated Jingmen First People's Hospital of Hubei Minzu University, Jingmen, \\ China \\ 2Department of Neurosurgery, The Second People's Hospital of Jingmen, Jingmen, China \\ ${ }^{3}$ Department of Oncology, The First Affiliated Hospital of Chongqing Medical University, Chongqing, China \\ *Equal contribution and share first authorship
}

Correspondence to: Ling Zhu; email: lingzhuVIP@yeah.net, https://orcid.org/0000-0002-1032-8478

Keywords: glioma, BMAL1, angiogenesis, HIF-1a, ANG2, VEGF

Received: February 26, $2021 \quad$ Accepted: November 11, $2021 \quad$ Published: November 23, 2021

Copyright: (C) 2021 Wang et al. This is an open access article distributed under the terms of the Creative Commons Attribution License (CC BY 3.0), which permits unrestricted use, distribution, and reproduction in any medium, provided the original author and source are credited.

\begin{abstract}
The rhythm gene BMAL1 (Brain and Muscle ARNT-Like 1) may play an important role in glioma tolerance for anti-angiogenesis therapy. In humans with glioma of different pathological grades, BMAL1 expression was significantly different, and the expression of ANG2 (Angiopoietin 2) and VEGF (Vascular endothelial growth factor) was positively correlated with the expression of BMAL1. Additionally, BMAL1 expression is positively correlated with the microvascular density and peritumoral edema of glioma. According to in vitro experiments, silencing the expression of BMAL1 in primary glioma cells results in a decrease in the expression of VEGF. In contrast, overexpression of BMAL1 promotes the expression of ANG2 and VEGF via HIF-1a pathway. Therefore, $B M A L 1$ likely participates in the angiogenesis of glioma by modulating ANG2 and VEGF expression, alters the therapeutic effect of anti-angiogenic treatments, and promotes peritumoral brain edema of glioma.
\end{abstract}

\section{INTRODUCTION}

Glioma is the most common malignancy of the central nervous system, and it is highly invasive, results in a poor prognosis, and is prone to recurrence after surgery. Although conventional treatment for glioblastoma involves extensive radical resection combined with adjuvant radiotherapy and chemotherapy, the one-year cumulative survival rate of patients is still less than $30 \%$. Only $3 \%$ of patients live longer than 5 years, with a total mean survival time of 6-9 months [1]. Therefore, studies aiming to determine the pathogenesis of glioma and identify methods to improve the clinical symptoms and prognosis of patients with glioma have become a hot topic in glioma research.

In recent years, vascular therapy targeting active angiogenic factors has become the standard treatment for some tumors, including colon cancer and kidney cancer $[2,3]$, and an adjuvant therapy for glioma $[4,5]$. As glioma is an angiogenesis-dependent tumor with many heterogeneous vessels in the tumor tissue [6], vascular targeted therapy has great potential in the treatment of glioma. However, for the treatment of intracranial tumors, single anti-vascular therapy is far from satisfactory in achieving a therapeutic effect similar to that observed in other tumors [7]. In addition to the limitations of the drug itself, the most important limitation is the drug resistance of tumor cells $[8,9]$. This tolerance may be related to rhythm genes [10].

In recent years, rhythm genes have been suggested to be related to the occurrence and development of various tumors, including breast cancer, colon cancer, and glioma [11-13]. As the core gene of rhythm gene, $B M A L 1$ is an important regulator that maintains normal cell and tissue homeostasis and plays an extremely critical role in tumor-related processes, such as cell 
proliferation and apoptosis, DNA repair, metabolism, and angiogenesis [14-17]. BMAL1 is also considered a pro-tumor factor in glioma that promotes the proliferation and migration of glioma cells [18]. Therefore, an examination of the regulatory effects of BMAL1 on the expression of the angiogenic factors $A N G 1$ (Angiopoietin 1), $A N G 2$, and $V E G F$ in glioma will be conducive to understanding the reasons for glioma tolerance to vascular targeted therapy and the development of new target drugs. HIF-la is an important regulatory factor of tumor angiogenesis. It was previously reported that $H I F-1 a$ can induce increased expression levels of Ang2 and $V E G F$ in hypoxia environment [19]. Therefore, in this study, we examined the correlation between the expression of the $B M A L 1$ gene and the expression of HIF-1a, ANG1, $A N G 2$, and $V E G F$ in human glioma tissues, as well as the relationships between the expression of the BMAL1 gene and the number of tumor microvessels and peritumor edema.

\section{RESULTS}

\section{Differential expression of $B M A L 1$ and $H I F-1 a$ in human glioma tissue}

BMAL1 was expressed at different levels in both glioma and normal tissues (non-tumor brain tissue specimens), and all the staining was observed in the nucleus (Figure 1). The expression of BMAL1 was detected in $74.68 \%(59 / 79)$ of glioma tissues, and 31 $(39.24 \%)$ of the 79 non-tumor brain tissues were positive for BMAL1. The expression level of BMAL1 in glioma cells was significantly different from nontumor brain cells $(P<0.05)$ (Table 1$)$.

BMAL1 expression was not different between WHO grade low-grade gliomas (I/II) and adjacent non-tumor tissues $(P>0.05)$. The BMAL1 was expressed at significantly higher levels in high-grade glioma (III/IV) than in low-grade glioma $(P<0.001)$ and adjacent non-tumor tissues $(P<0.001)$ (Table 1). In addition, BMAL1 expression did not display significant relationships with age, gender, or tumor size (Table 2).

Differential expression of HIF-1a was also observed between tumor tissues and peri-tumor tissues, and the positive expression was mainly concentrated in the nucleus (Figure 1). The expression of HIF-1a in highgrade gliomas was significantly higher than that in adjacent peritumor tissues $(P<0.001)$ and low-grade glioma $(P<0.01)$. Different from BMAL1 expression, HIF-1a expression in low-grade glioma tissues differs from that in adjacent peritumor tissues $(P<0.05)$.
Expression of the angiogenesis-related genes ANG1, ANG2 and VEGF in human glioma tissues of different WHO pathological grades

In glioma tissue, ANG1, ANG2, and VEGF are expressed in vascular endothelial cells and tumor cells (Figure 1) (Table 3). A significant difference in ANG1 expression was not observed between tumor tissues (32/79) and non-tumor tissues (34/79) $(P>0.05)$. However, significantly higher ANG1 expression was detected in grade I/II (23/38) glioma tissues $(P<0.001)$ than in grade III/IV $(9 / 41)$ tumors.

The expression of ANG2 in glioma tissue (48/79) and non-tumor tissue $(15 / 79)$ was significantly different $(P<0.05)$. Moreover, this difference was also observed in glioma tissues of different pathological grades. A significant difference in ANG2 expression was observed between grade I/II glioma (10/38) and grade III/IV glioma $(38 / 41)(P<0.001)$.

The expression of VEGF in glioma tissue was similar to Ang2, and a significant difference $(P<0.05)$ was observed between tumor tissues (59/79) and peritumor tissues (22/79). VEGF expression in grade III/IV tissues (36/41) was different from its expression in grade I/II glioma tissue $(23 / 38)(P<0.05)$.

The correlation between BMAL1 expression and the expression of ANG1, ANG2, VEGF and HIF-1a

In this study, $17.72 \%(14 / 79)$ of samples were $\mathrm{BMAL}^{-} / \mathrm{ANG}^{+}$, a significant difference was observed compared with $\mathrm{BMAL1}^{+} / \mathrm{ANG}^{+}$samples, $53.16 \%(42 / 79)(P<0.05)$, and the expression of the two was negatively correlated $(\mathrm{R}=-0.365, P<$ $0.001)$. Notably, $55.7 \%$ (44/79) of samples were $\mathrm{BMAL}^{+} / \mathrm{ANG}^{+}$, which was significantly higher than the percentage of $\mathrm{BMAL1}^{-} / \mathrm{ANG}^{+}$samples $(7.59 \%, 6 / 79)$, and the difference was significant $(P<$ $0.05)$; a significant positive correlation was observed between the two ( $\mathrm{R}=0.402, P<0.001) .63 .29 \%$ $(50 / 79)$ of samples were $\mathrm{BMAL1}^{+} / \mathrm{VEGF}^{+}$, a value that is significantly higher than the $11.39 \%(9 / 79)$ of $\mathrm{BMAL1}^{-} / \mathrm{VEGF}^{+}$samples, and the two were positively correlated $(\mathrm{R}=0.397, P<0.001)$. There was a significant difference between BMAL1 ${ }^{-} / \mathrm{HIF}$ $1 \mathrm{a}^{+}$samples $(13.92 \%, 11 / 79)$ and $\mathrm{BMAL1}^{+} / \mathrm{HIF}-1 \mathrm{a}^{+}$ samples $(70.88 \%, 56 / 79)(P<0.01)$, and the two were also positively correlated $(\mathrm{R}=0.675, P<$ 0.001). These data indicated that increased expression of BMAL1 may promote the expression of HIF-1a, ANG2 and VEGF in glioma (Figure 2A). 
Table 1. Comparison of BMAL1 positive expression between glioma and peritumoral tissues, and between different pathological grades and peritumoral tissues.

\begin{tabular}{lccc}
\hline \multicolumn{1}{c}{ Group } & $\boldsymbol{n}$ & BMAL1 Positive & $\boldsymbol{P}$ \\
\hline Tumoral tissues & 79 & $59(74.68 \%)$ & $P<0.05$ \\
Peritumor tissue & 79 & $39(39.24 \%)$ & \\
I/II grades & 38 & $20(52.63 \%)$ & $P=0.491>0.05$ \\
Peritumoral tissues around I/II grades & 38 & $17(44.74 \%)$ & \\
III/IV grades & 41 & $39(95.12 \%)$ & $P<0.001$ \\
Peritumoral tissues around III/IV grades & 41 & $22(53.66 \%)$ & \\
I/II grades & 38 & $20(52.63 \%)$ & $P<0.001$ \\
III/IV grades & 41 & $39(95.12 \%)$ & \\
\hline
\end{tabular}
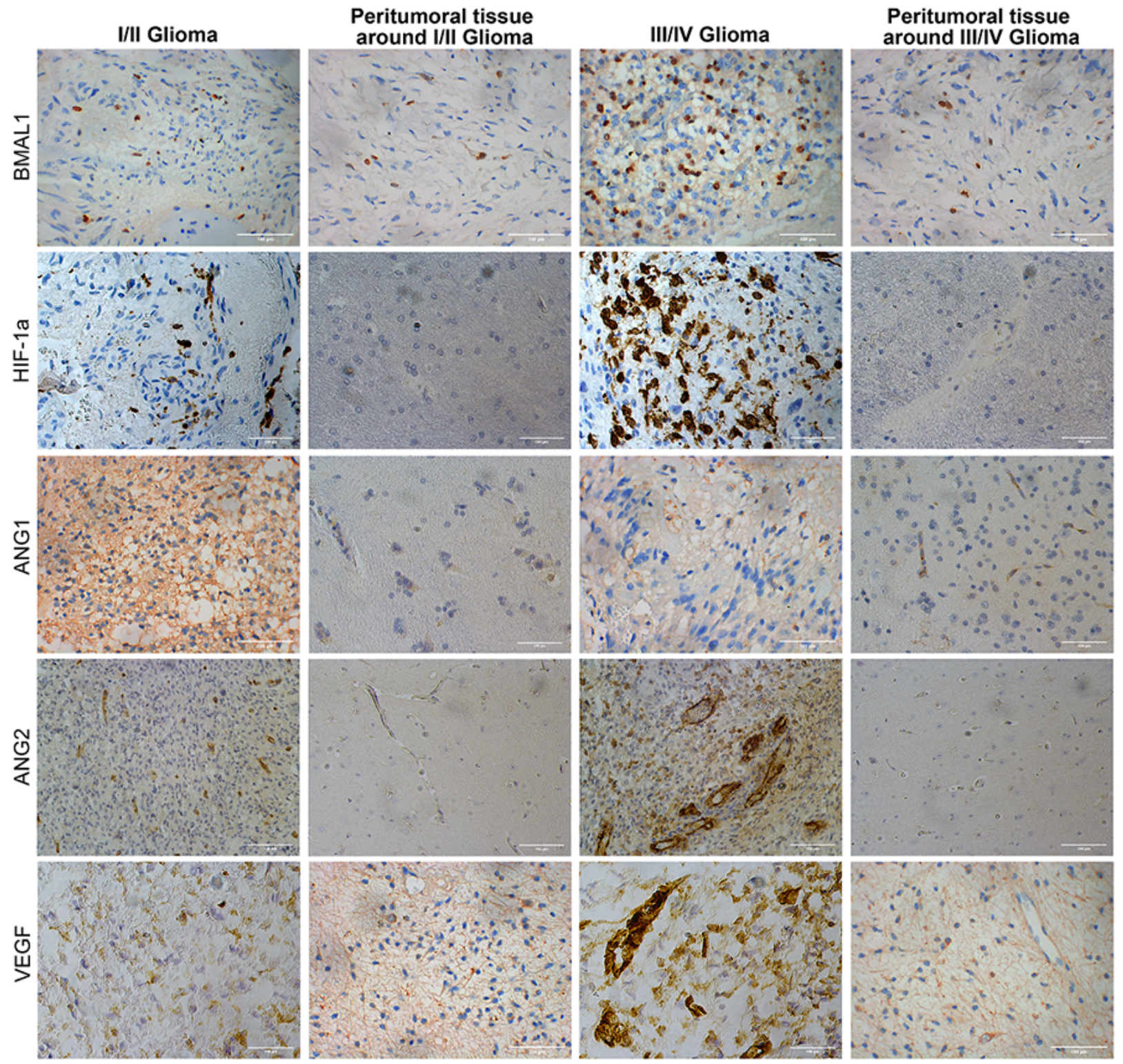

Figure 1. Immunohistochemistry analysis of BMAL1, HIF-1a, ANG1, ANG2, and VEGF expression in glioma and normal tissues with different pathological grades. 
Table 2. Relationship between BAML1 expression and clinical data related to glioma.

\begin{tabular}{llrl}
\hline \multicolumn{1}{c}{ Sample } & $\boldsymbol{n}$ & BMAL1 Positive & $\boldsymbol{P}$ \\
\hline Age & & $25(73.53 \%)$ & \\
$<40$ & 34 & $34(75.56 \%)$ & $P=0.819>0.05$ \\
$>40$ & 45 & $31(73.81 \%)$ & \\
Sexual & 42 & $28(75.68 \%)$ & $P=0.827>0.05$ \\
Male & 37 & $34(75.56 \%)$ & \\
Female & 45 & $25(73.53 \%)$ & $P=1.000>0.05$ \\
Tumor volume & 34 & & \\
$>4 \mathrm{~cm}$ & &
\end{tabular}

\section{Correlation between expression and the tumor microvascular density}

CD34 was used to label the endothelial cells in tumor tissues and determine the microvascular density. The MVD (Microvascular density) was measured in the tumor using the Weidner method [20]. In gliomas, the MVD of BMAL1 ${ }^{-}$samples was $25.3 \pm 10.93$ and the MVD of and BMAL1 ${ }^{+}$samples was $43.75 \pm 10.94$, with a statistically significant difference $(P<0.05)$ (Figure $2 \mathrm{~B}$ and $2 \mathrm{C})$. In gliomas, BMAL1 expression was significantly and positively correlated with the tumor MVD ( $\mathrm{R}=0.915, P<0.001)$, suggesting that BMAL1 is closely related to angiogenesis in glioma (Table 4).

\section{Correlation of BMAL1 expression with clinical cerebral edema}

According to the EI (edema index), we performed peritumor cerebral edema grading in 79 patients with glioma [21]. The median EI was 4.79 (range, 1.2010.52): 0 with no edema, $16(20.3 \%)$ with mild edema, $26(32.9 \%)$ with moderate edema, and $37(46.8 \%)$ with severe edema (Table 5). Although clinical MRI scans suggested that all patients had peritumor brain edema to varying degrees, compared with the $\mathrm{BMAL1}^{-}$patients, patients with $\mathrm{BMAL1}^{+}$expression were more likely to exhibit moderately severe or severe brain edema (Figure $3 \mathrm{~A}$ and $3 \mathrm{~B}$ ). Furthermore, we analyzed the BMAL1 levels
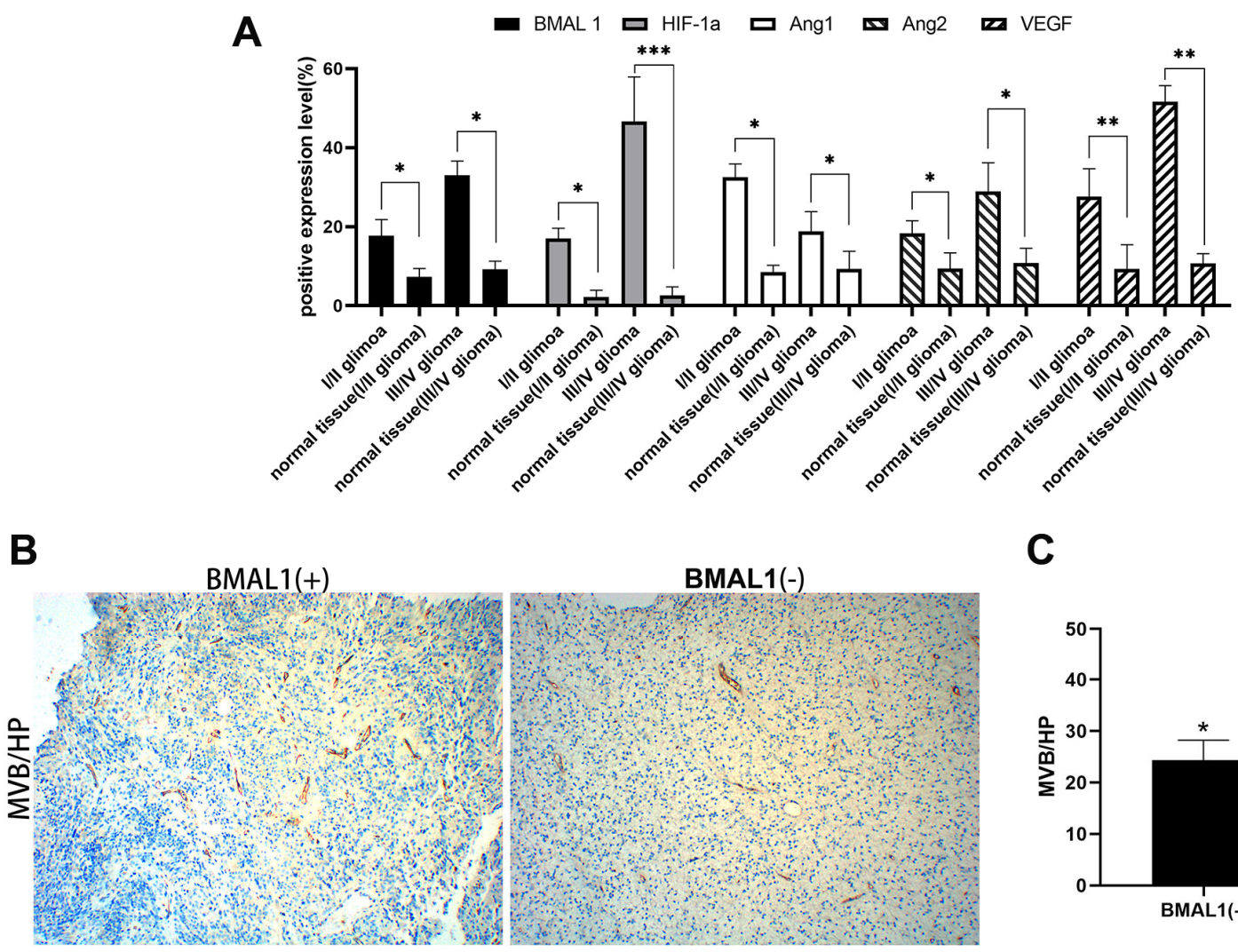

C

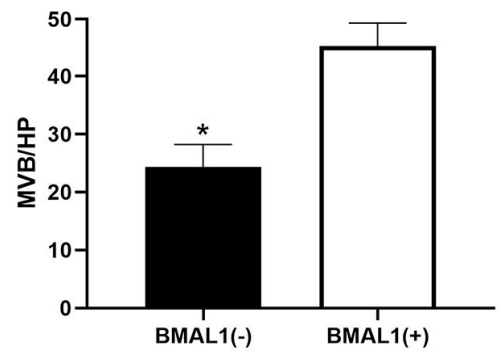

Figure 2. Expression analysis of BMAL1 and CD34. (A) ANOVA test was used for comparisons the expression of BMAL1 in glioma and normal tissues with different pathological grades. (B) A set of representative graphs displaying the number of microvessels in gliomas by labeling CD34. (C) Two-sample $t$-test was used for comparisons between the two groups. ${ }^{*} p<0.05,{ }^{* *} p<0.01 .{ }^{* * *} p<0.001$. 
Table 3. Relationship between expression of ANG 1, ANG 2, VEGF and pathological features of glioma.

\begin{tabular}{|c|c|c|c|}
\hline Sample & $N$ & Positive rate & $P$ \\
\hline \multicolumn{4}{|l|}{ ANG1 } \\
\hline Peritumoral tissues & 79 & $34(43.04 \%)$ & \\
\hline Tumor & 79 & $32(40.51 \%)$ & $P=0.747>0.05$ \\
\hline \multicolumn{4}{|l|}{ ANG1 } \\
\hline I/II grades & 38 & $23(60.35 \%)$ & \\
\hline III/IV grades & 41 & $9(21.95 \%)$ & $P<0.01$ \\
\hline \multicolumn{4}{|l|}{ ANG2 } \\
\hline Peritumoral tissues & 79 & $15(18.99 \%)$ & \\
\hline Tumor & 79 & $49(62.03 \%)$ & $P<0.01$ \\
\hline \multicolumn{4}{|l|}{ ANG2 } \\
\hline I/II grades & 38 & $11(28.95 \%)$ & \\
\hline III/IV grades & 41 & $38(92.68 \%)$ & $P<0.01$ \\
\hline \multicolumn{4}{|l|}{ VEGF } \\
\hline Peritumoral tissues & 79 & $22(27.85 \%)$ & \\
\hline Tumor & 79 & $59(74.68 \%)$ & $P<0.01$ \\
\hline \multicolumn{4}{|l|}{ VEGF } \\
\hline I/II grades & 38 & $23(60.53 \%)$ & \\
\hline III/IV grades & 41 & $36(87.80 \%)$ & $P<0.05$ \\
\hline
\end{tabular}
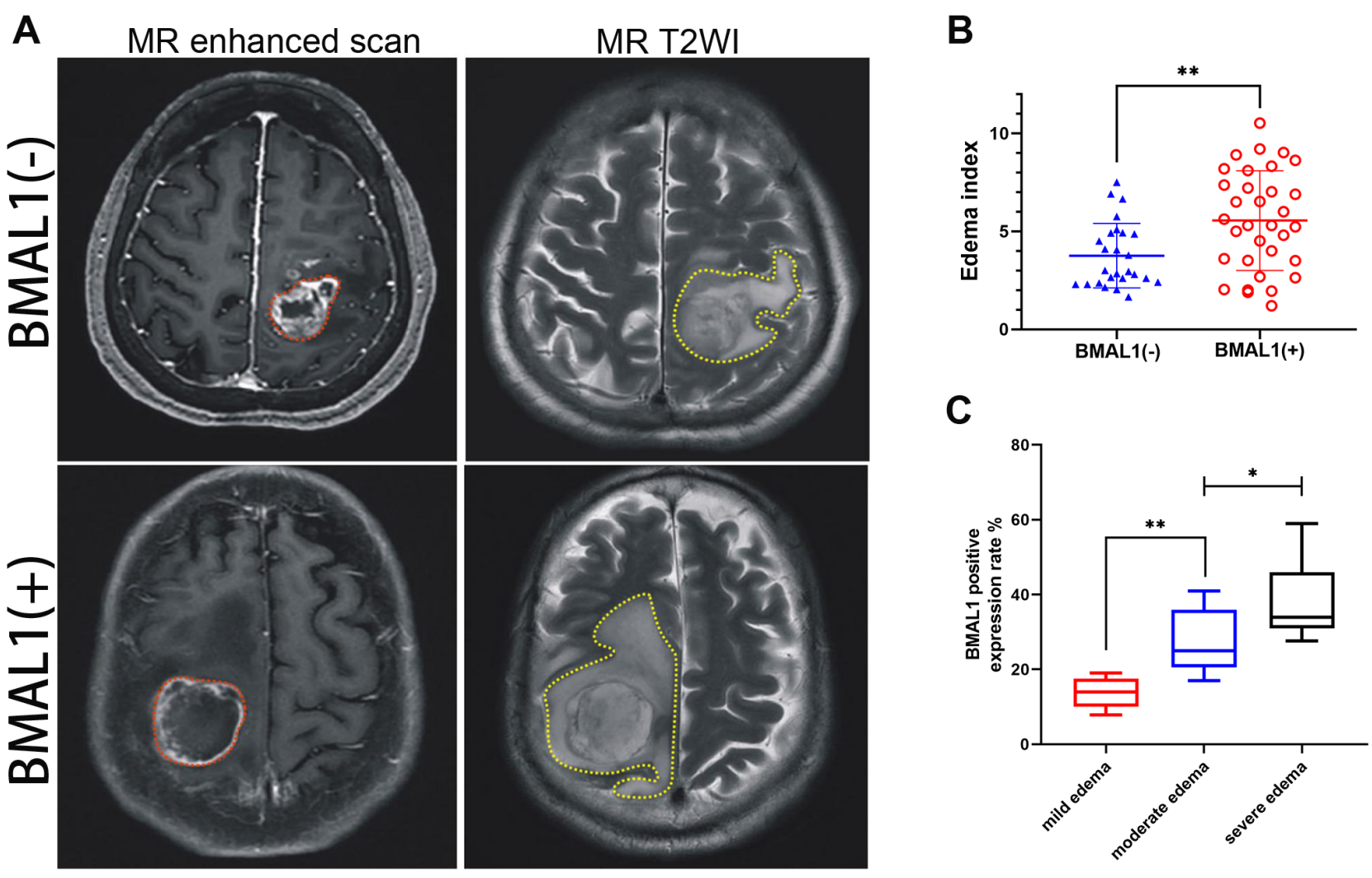

Figure 3. MR images of clinical patients showed the relationship between peritumoral brain edema and BMAL1 expression. (A) A set of representative graphs displaying MR images of brain edema. The red dotted line is the solid part of the tumor, and the yellow dotted line is the area of brain edema around the tumor. Patients with BMAL1 (+) had obvious peritumoral edema. (B) The El was compared between the BAML1 (+) expression group $(n=59)$ and the BAML1 $(-)$ expression group $(n=20)$. (C) BMAL1 expression levels in the mild edema group $(n=5)$, moderate edema group $(n=15)$, and severe edema group $(n=39)$ were assessed. ${ }^{*} p<0.05,{ }^{* * *} p<0.01 .{ }^{* * *} p<$ 0.001 . 
Table 4. Correlation between BMAL1 expression and MVD.

\begin{tabular}{ccccc}
\hline BMAL1 Expression & $\boldsymbol{n}$ & MVD (+)/HP & R & $\boldsymbol{P}$ \\
\hline- & 20 & $25.3 \pm 10.93$ & & \\
+ & 59 & $43.75 \pm 10.94$ & 0.915 & $P<0.001$ \\
\hline
\end{tabular}

Notes: HP: high power field.

Table 5. Correlation between BMAL1 expression and edema.

\begin{tabular}{cccrcc}
\hline BMAL1 & $\boldsymbol{n}$ & + & Edema & $\mathbf{X}^{2}$ & $\boldsymbol{P}$ \\
\hline Expression & 59 & 5 & 54 & & \\
- & 11 & 9 & 20.02 & $<0.0001$ \\
\hline
\end{tabular}

Notes: +: mild edema $(1<\mathrm{EI} \leq 1.5) ;++$ : moderate edema $(1.5<\mathrm{EI} \leq 3) ;+++$ : severe edema $(\mathrm{EI}>3)$.

in different edema groups. The BMAL1 expression level in the moderate edema group was higher than that in the mild edema group $(P<0.01)$, and the expression level in the severe edema group was higher than that in moderate edema group $(P<0.05)$ (Figure 3C).

\section{The regulatory effect of BMAL1 expression on HIF-1a, ANG1, ANG2 and VEGF expression}

After the silencing of the BMAL1, the expression of VEGF and HIF-1a were significantly decreased in the BMAL1-Sh group compared with the BMAL1-NC group and the BMAL1-Sh NC group (Figure 4A and 4B); however, a similar result did not found in the expression of ANG2, compared with the BMAL1-NC group $(P=0.156>0.05)$ and the BMAL1-Sh NC group $(P=0.239>0.05)$; a significant difference in ANG1 expression was not observed compared with the BMAL1-NC group $(P=0.193>0.05)$ and BMAL1-Sh NC group $(P=0.218>0.05)$. A subtle difference expression pattern was observed in glioma cells that overexpressed BMAL1. In other words, after the overexpression of the BMAL1 gene, the expression of HIF-1a, ANG2 and VEGF was correspondingly increased compared with the BMAL1-NC group and the BMAL1-SHNC group (Figure 4A and 4B). However, no difference in expression of ANG1 was observed compared with the BMAL1-NC group $(P=0.203>$ $0.05)$ and the BMAL1-SHNC group $(P=0.114>0.05)$.

\section{DISCUSSION}

Glioma is considered an angiogenesis-dependent tumor [22]. A serious imbalance between vascular growthpromoting and inhibitory factors in the glioma microenvironment has been observed [23]. As previously reported, $A N G 1, A N G 2$ and $V E G F$ are expressed at high levels in gliomas, and significant differences in their expression were observed in different pathological grades. Therefore, anti- angiogenic therapy for gliomas should be promising; however, in fact, glioma is one of the tumors in which anti-angiogenesis therapy displays poor efficacy [9]. Based on these findings, a single target therapy targeting a specific vascular factor may not improve the angiogenesis and vascular structure of glioma, or other more complex regulatory mechanisms may regulate angiogenesis in glioma.

High BMAL1 expression in tumor cells was associated with poor clinical outcomes in patients with colorectal cancer receiving Beva (anti-VEGF) combined with chemotherapy [10]. Furthermore, this resistance to Beva was caused by an internal, intracellular "escape" pathway of the tumor cells that involved BMAL1. This pathway initiates the continuous synthesis and secretion of VEGF, followed by continued stimulation of the VEGFA/VEGFR2 signaling pathway through autocrine/paracrine mechanisms [10]. Lasse et al. also confirmed that BMAL1 regulated angiogenesis through the $V E G F$ pathway in zebrafish [17]. Thus, rhythm genes may contribute to glioma tolerance to vascular targeted therapy. In addition to $V E G F, B M A L 1$ was also positively correlated with the expression of $A N G 2$ in glioma in our study. It has been demonstrated that the hypoxic microenvironment of glioma could upregulate the expression of $A N G 2$ and $V E G F$ via $H I F$ la pathway $[19,24]$. The above-mentioned findings prompted us to investigate the correlation between HIF-1a and BMAL1 expression in glioma. Our IHC results showed that the intensity of HIF-1a positive expression was positively correlated with BMAL1 expression. Additionally, the expression of BMAL1 correlated with the pathological grade of glioma, but not with the patient's age, gender, or tumor size. Therefore, BMAL1 expression may be independent of the initial origin of glioma, but is related to the later development of glioma, and BMAL1 is likely to participate in the mechanism regulating glioma angiogenesis and we hypothesized that BMAL1 may 
up-regulate the expression of $V E G F$ and $A N G 2$ through the $H I F-1 a$ pathway.

To more directly assess the impact of BMAL1 on the expression levels of $H I F-1 a, A N G 2$ and $V E G F$ we used primary glioma cells to construct cell models with $B M A L 1$ overexpression and silencing and to determine whether BMAL1 is involved in $H I F-1 a, A N G 2$ and $V E G F$ expression in gliomas. In our study, when the $B M A L 1$ was up-regulated, $H I F-1 a, A N G 2$ and $V E G F$ expression also increased; when $B M A L 1$ expression was silenced, $H I F-1 a$ and $V E G F$ expression decreased, while the expression of $A N G 1, A N G 2$ was not affected. It is a fascinating result that $H I F-1 a, V E G F$ increases or decreases with the increase or silencing of $B M A L 1$ expression. Thus, BMAL1 may be involved in regulating the expression of $V E G F, H I F-1 a$. This result is consistent with the findings reported by Lasse and colleagues who found that the $V E G F$ expression pattern matched the expression pattern of BMAL1 and BMAL1
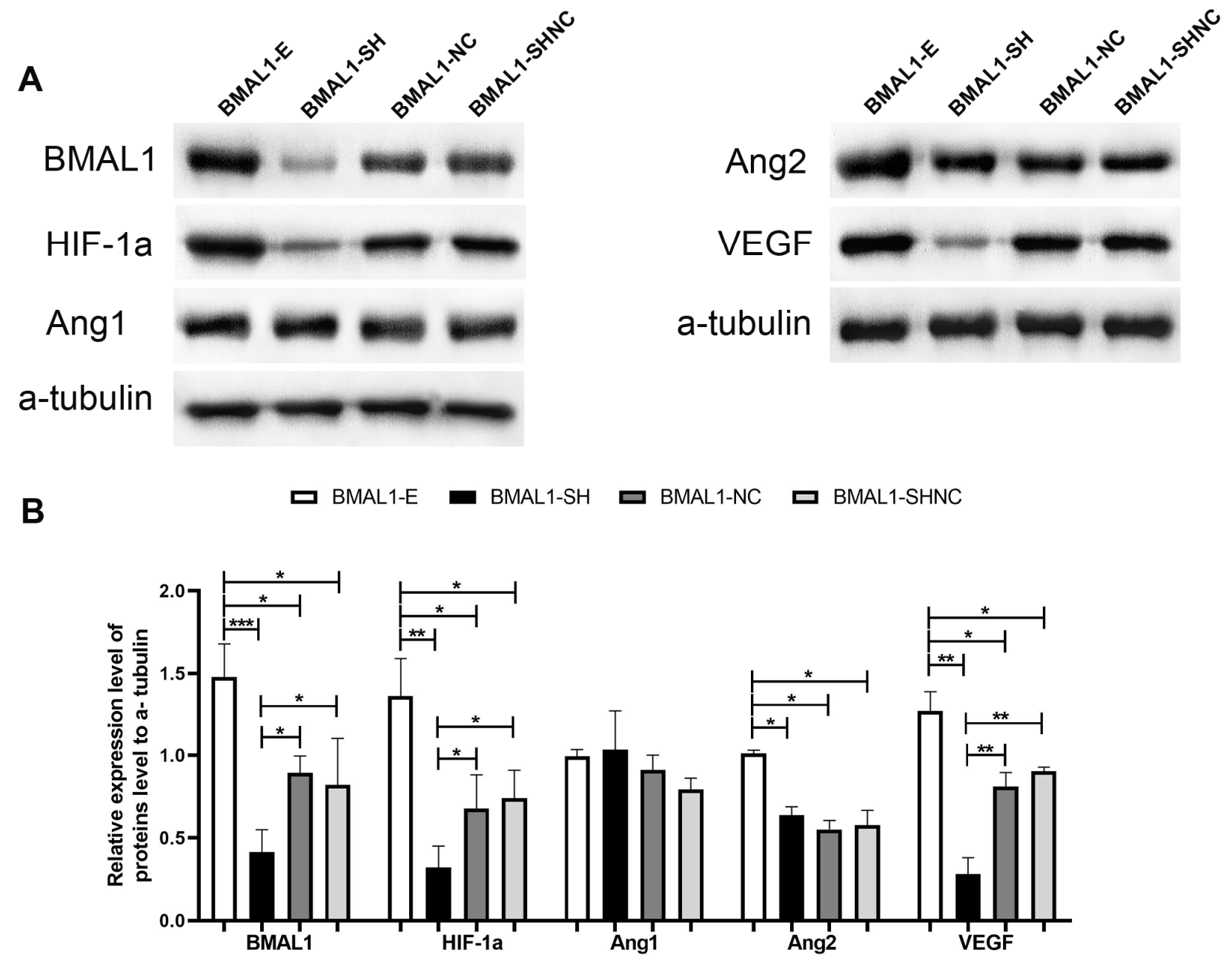

Figure 4. The regulatory pathway of BMAL1 on proangiogenic factors was assessed by western blot. (A) A set of representative graphs displaying the regulatory pathway of BMAL1 in glioma cells. (B) Two-sample $t$-test was used for comparisons between the two groups. ${ }^{*} p<0.05,{ }^{* *} p<0.01 .{ }^{* * *} p<0.001$. 
heterogeneous blood vessels in tumor tissues [26]. Dual antagonists targeting ANG2/VEGF effectively improve the vascular structure and function and reduce peritumor brain edema in patients with glioma [25, 27]. In the present study, BMAL1 expression was positively correlated with the expression of $A N G 2$ and $V E G F$, and thus we speculated that high BMAL1 expression might be correlated with the microvascular density of glioma and the degree of peritumor brain edema. We verified our hypothesis by analyzing human glioma tissue to determine the correlation between BMAL1 expression and microvessels, and performed MRI (Magnetic Resonance Imaging) to understand the correlation between the expression of the BMAL1 gene and peritumor edema. The positive expression of BMAL1 protein in tumor tissues correlated with the distribution of high MVD, which was more obvious in high-grade glioma. Similar results were also obtained using MRI to assess brain edema; the higher the positive rate of $B M A L 1$ expression, the more severe the brain edema. Based on these results, BMAL1 may plays an important role in brain tumor microangiogenesis and peritumor edema.

In summary, we speculated that BMAL1 might be involved in angiogenesis in human glioma cells by regulating $V E G F$ and $A N G 2$ expression via $H I F-l a$ pathway. Researchers have not determined whether BMAL1 regulates tumor biological behaviors by regulating angiogenesis in glioma. However, our results support the hypothesis that BMAL1 is involved in glioma angiogenesis by regulating the ANG2/VEGF pathway and affects the degree of peritumor brain edema. Although the specific molecular pathway is not yet clear, this discovery will be beneficial to developing new clinical drugs for glioma and obtaining a better understanding of the mechanism underlying the development of peritumor edema, providing new insights into the treatment of peritumor brain edema.

\section{MATERIALS AND METHODS}

\section{Clinical samples}

All the specimens were collected at the Neurosurgery Department of Jingmen First People's Hospital affiliated with Hubei Minzu University from January 2017 to October 2020. None of the patients had received preoperative radiotherapy or chemotherapy. The specimens were removed from 12:00 noon to before 3:00 p.m., and 79 glioma specimens were collected. The patients were 17-69 years old. The patients included 42 males and 37 females. According to WHO glioma grading criteria, 38 cases of low-grade astrocytoma (grade I/II) and 41 cases (grade III/IV) of anaplastic astrocytoma and glioblastoma multiforme were obtained. Nonneoplastic tissue samples were collected from 79 patients. Some of the specimens were prepared as paraffin sections for immunohistochemistry, and primary glioma cells were extracted from other specimens within one hour.

\section{Primary glioma cell isolation and culture}

Within an hour of obtaining fresh specimens of glioma tissues (WHO class III/IV), the samples were transferred on ice to the ultra-clean cabinet, minced, rinsed with saline repeatedly, centrifuged, and the supernatant was digested with II collagenase at $37^{\circ} \mathrm{C}$ until most of the tissue block was dissociated. DMEM containing 15\% fetal bovine serum (FBS) was added to terminate the digestion, cells were filtered through a $70 \mu \mathrm{m}$ cell mesh filter, centrifuged, the supernatant was discarded, and pellets were resuspended and cultured in DMEM.

\section{MRI examination and edema index measurement}

Patients underwent an MRI plain scan and contrastenhanced scan before the operation with a Siemens 3.0 T MR machine. The protocol of Qu et al. [19] was used to examine MRI and measure the edema index. All MR imaging data were measured by a computer workstation. Preoperative T1-enhanced TSE sequence of MRI was employed to measure tumor volume. Specifically, the first step was to measure the axial maximum diameter (A), coronal maximum diameter (B), and sagittal maximum diameter $(\mathrm{C})$ of the tumor on MR imaging T1enhanced TSE sequence $(\mathrm{TR}=2000 \mathrm{~ms}$; $\mathrm{TE}=9 \mathrm{~ms}$; section thickness $=6 \mathrm{~mm}$; FOV $=230 \times 187 \mathrm{~mm}$; voxel size $=0.7 \times 0.7 \times 6.0 \mathrm{~mm}$ ) in each patient. The second step is to calculate the tumor volume according to the formula previously reported: $\mathrm{V}=4 / 3 \pi \times \mathrm{A} / 2 \times \mathrm{B} / 2 \times \mathrm{C} / 2$ [28]. The volume of perituminal brain edema was measured using T2-weighted and FLAIR TSE sequences, with specific MRI operating parameters as follows: TR = $9000 \mathrm{~ms}$; TE $=81 \mathrm{~ms}$; section thickness $=6 \mathrm{~mm}$; FOV $=$ $230 \times 200 \mathrm{~mm}$; voxel size $=0.7 \times 0.7 \times 6.0 \mathrm{~mm}$. As well as measuring tumor volume, we measured the maximum diameter of cerebral edema in axial, coronal, and sagittal scans and calculated the edema volume. The edema index (EI) was used to evaluate the degree of peritumoral edema, and its calculation formula was as follows: $\mathrm{EI}=$ $(\mathrm{V}$ tumor + edema $) /(\mathrm{V}$ tumor $)$. According to previous reports, peritumoral edema can be divided into four degrees according to the edema index [29], as follow: no edema $(E I=1)$, mild edema $(1<\mathrm{EI} \leq 1.5)$, moderate edema $(1.5<\mathrm{EI} \leq 3)$, and severe edema $(\mathrm{EI}>3)$.

\section{Immunohistochemistry}

Immunohistochemistry (IHC) was performed using the SP method. The specimens were prepared as paraffin 
sections, baked, dewaxed and hydrated, subjected to dehydration with a gradient of ethanol solution, and subjected to the blocking of endogenous peroxidase activity, antigen repair (high-pressure repair), washing, sealing, and an incubation with BMAL1 (ab231793, ABCAM), HIF-1a (ab114977, ABCAM), ANG1 (ab133425, ABCAM), ANG2 (Ab155106, ABCAM), VEGF (ab32152, ABCAM) and CD34 (ab8158, ABCAM) primary antibodies at $4^{\circ} \mathrm{C}$ overnight. Next, sections were incubated with secondary antibodies, DAB was used for color development, and sections were stained with hematoxylin, dehydrated, sealed, and observed under a microscope.

\section{Construction of BMAL1 overexpression and silencing vectors}

Synthetic primers were designed based on the BMAL 1 gene sequence in NCBI. The following primer sequences were used: upstream lead 5'-CCGGAAT TCGCCACCATGGCAGACCAGAGAATGGACATTT CTTC-3; downstream primers: 5'-CCGGGATCCT TACAGCGGCCATGGCAAGTC-3'. Under the action of T4 DNA ligase, the lentivirus vector PGMLV-PA6 was connected to the BMAL1 fragment.

Based on the sequence of the BMAL1 gene, a synthetic shRNA oligomeric single-stranded DNA with the following sequence $\left(5^{\prime}\right.$ to $\left.3^{\prime}\right)$ designed: BMAL1 sh3T: GATCCGCACGCGATAGATGGAAAGTTCTCGAGA ACTTTCCATCTATCGCGTGCTTTTTT and BMAL1 sh3B: AATTAAAAAAGCACGCGATAGATGGAA AGTTCTCGAGAACTTTCCATCTATCGCGTGCG. The oligomers were annealed to form a double-stranded structure. Under the action of T4 DNA ligase, the lentivirus vector PGMLV-SB3 was connected with the double-stranded primer. The following steps were conducted according to the manufacturer's instructions and products were stored at $-80^{\circ} \mathrm{C}$.

\section{Transfection and western blotting}

The virus stock was diluted to an appropriate MOI (multiplicity of infection), and the diluted lentivirus was added to the primary cultured cells. Puromycin was used to screen stably transfected cells. Finally, the following four groups were obtained: the BMAL1 overexpression group (BMAL1-E), BMAL1 overexpression control group (BMAL1-NC), BMAL1 silent expression group (BMAL1-SH), and BMAL1 silent expression control group (BMAL1-SHNC). Cells from these experimental groups were cultured in 6 well plate. After reaching $70 \%$ confluence, the cells were lysed on ice using RIPA buffer. After protein extraction, the protein samples were loaded in the wells of SDSPAGE gels and separated. The expression of target proteins BMAL1 (ab231793, ABCAM), HIF-1a (ab114977, ABCAM), ANG1 (ab133425, ABCAM), ANG2 (Ab155106, ABCAM) and VEGF (ab32152, ABCAM) was detected by Western blot.

\section{Ethics statement for human specimens}

All studies on human subjects were approved by Hubei University for Nationalities Ethics Committee. The ethical approval number for excised material is HBMZ2012018.

\section{Statistical analysis}

The association between tumor grade (high-grade/lowgrade gliomas) and expression of the investigated proteins (negative/positive) was assessed using the ChiSquare Test and Two-sample $t$-test, included in the Statistical Package for the Social Science, version 13.0. Using Kendall's tau-b Correlation to analyse correlation between the expression of BMAL1 and ANG1, ANG2, VEGF and edema index. Using Spearman Correlation to analyse correlation between the expression of BMAL1 and MVD.

\section{Data availability}

The datasets generated during and/or analysed during the current study are available from the corresponding author on reasonable request.

\section{AUTHOR CONTRIBUTIONS}

Fan Wan: Conceptualization, Methodology, Software. CaiYan Li: Data curation, Writing- Original draft preparation. Fei Han: cell culture, Construction of lentiviral vectors. LvAn Chen: MR, ICH, Sample collection. Ling Zhu: Data analysis, paper modification.

\section{CONFLICTS OF INTEREST}

We declare that we have no financial and personal relationships with other people or organizations that can inappropriately influence our work, there is no professional or other personal interest of any nature or kind in any product, service and/or company that could be construed as influencing the position presented in, or the review of, the manuscript entitled.

\section{FUNDING}

This research was supported by the Natural Science Foundation of HuBei Province of China (Grant 2020CFB542); Hubei Provincial Medical Youth Topnotch Talents Project. 


\section{REFERENCES}

1. Louis DN, Perry A, Reifenberger G, von Deimling A, Figarella-Branger D, Cavenee WK, Ohgaki H, Wiestler OD, Kleihues P, Ellison DW. The 2016 World Health Organization Classification of Tumors of the Central Nervous System: a summary. Acta Neuropathol. 2016; 131:803-20.

https://doi.org/10.1007/s00401-016-1545-1 PMID:27157931

2. Bottsford-Miller JN, Coleman RL, Sood AK. Resistance and escape from antiangiogenesis therapy: clinical implications and future strategies. J Clin Oncol. 2012; 30:4026-34.

https://doi.org/10.1200/JCO.2012.41.9242 PMID:23008289

3. Jayson GC, Hicklin DJ, Ellis LM. Antiangiogenic therapy--evolving view based on clinical trial results. Nat Rev Clin Oncol. 2012; 9:297-303. https://doi.org/10.1038/nrclinonc.2012.8 PMID:22330688

4. Sorensen AG, Batchelor TT, Zhang WT, Chen PJ, Yeo P, Wang $M$, Jennings $D$, Wen PY, Lahdenranta J, Ancukiewicz M, di Tomaso E, Duda DG, Jain RK. A "vascular normalization index" as potential mechanistic biomarker to predict survival after a single dose of cediranib in recurrent glioblastoma patients. Cancer Res. 2009; 69:5296-300. https://doi.org/10.1158/0008-5472.CAN-09-0814 PMID:19549889

5. Jain RK. Normalizing tumor vasculature with antiangiogenic therapy: a new paradigm for combination therapy. Nat Med. 2001; 7:987-89. https://doi.org/10.1038/nm0901-987 PMID:11533692

6. Griscelli $\mathrm{F}$, Li H, Cheong C, Opolon P, BennaceurGriscelli A, Vassal G, Soria J, Soria C, Lu H, Perricaudet $M$, Yeh P. Combined effects of radiotherapy and angiostatin gene therapy in glioma tumor model. Proc Natl Acad Sci U S A. 2000; 97:6698-703.

https://doi.org/10.1073/pnas.110134297 PMID:10823901

7. Miletic H, Niclou SP, Johansson M, Bjerkvig R. AntiVEGF therapies for malignant glioma: treatment effects and escape mechanisms. Expert Opin Ther Targets. 2009; 13:455-68. https://doi.org/10.1517/14728220902806444 PMID:19335067

8. Osswald M, Jung E, Sahm F, Solecki G, Venkataramani V, Blaes J, Weil S, Horstmann H, Wiestler B, Syed M, Huang L, Ratliff $M$, Karimian Jazi K, et al. Brain tumour cells interconnect to a functional and resistant network. Nature. 2015; 528:93-98. https://doi.org/10.1038/nature16071

PMID:26536111

9. Wick W, Platten $M$, Wick $A$, Hertenstein A, Radbruch $A$, Bendszus $M$, Winkler F. Current status and future directions of anti-angiogenic therapy for gliomas. Neuro Oncol. 2016; 18:315-28. https://doi.org/10.1093/neuonc/nov180 PMID:26459812

10. Burgermeister E, Battaglin F, Eladly F, Wu W, Herweck F, Schulte N, Betge J, Härtel N, Kather JN, Weis CA, Gaiser T, Marx A, Weiss C, et al. Aryl hydrocarbon receptor nuclear translocator-like (ARNTL/BMAL1) is associated with bevacizumab resistance in colorectal cancer via regulation of vascular endothelial growth factor $A$. EBioMedicine. 2019; 45:139-54.

https://doi.org/10.1016/i.ebiom.2019.07.004 PMID:31300350

11. Reszka E, Przybek M, Muurlink O, Pepłonska B. Circadian gene variants and breast cancer. Cancer Lett. 2017; 390:137-45.

https://doi.org/10.1016/i.canlet.2017.01.012 PMID:28109907

12. Okazaki F, Matsunaga N, Okazaki H, Azuma H, Hamamura K, Tsuruta A, Tsurudome $Y$, Ogino T, Hara $Y$, Suzuki T, Hyodo K, Ishihara H, Kikuchi $H$, et al. Circadian Clock in a Mouse Colon Tumor Regulates Intracellular Iron Levels to Promote Tumor Progression. J Biol Chem. 2016; 291:7017-28. https://doi.org/10.1074/ibc.M115.713412 PMID:26797126

13. Khan $S$, Liu $Y$, Siddique $R$, Nabi G, Xue M, Hou $H$. Impact of chronically alternating light-dark cycles on circadian clock mediated expression of cancer (glioma)-related genes in the brain. Int J Biol Sci. 2019; 15:1816-34.

https://doi.org/10.7150/ijbs.35520

PMID:31523185

14. Sulli G, Lam MTY, Panda S. Interplay between Circadian Clock and Cancer: New Frontiers for Cancer Treatment. Trends Cancer. 2019; 5:475-94. https://doi.org/10.1016/i.trecan.2019.07.002 PMID:31421905

15. Masri S, Sassone-Corsi P. The emerging link between cancer, metabolism, and circadian rhythms. Nat Med. 2018; 24:1795-803.

https://doi.org/10.1038/s41591-018-0271-8 PMID:30523327

16. Puram RV, Kowalczyk MS, de Boer CG, Schneider RK, Miller PG, McConkey M, Tothova Z, Tejero H, Heckl D, Järås $\mathrm{M}$, Chen $\mathrm{MC}$, Li $\mathrm{H}$, Tamayo $\mathrm{A}$, et al. Core Circadian Clock Genes Regulate Leukemia Stem Cells in AML. Cell. 2016; 165:303-16. 
https://doi.org/10.1016/j.cell.2016.03.015

PMID:27058663

17. Jensen LD, Cao Y. Clock controls angiogenesis. Cell Cycle. 2013; 12:405-408.

https://doi.org/10.4161/cc.23596

PMID:23324349

18. Li A, Lin X, Tan X, Yin B, Han W, Zhao J, Yuan J, Qiang $B$, Peng $X$. Circadian gene Clock contributes to cell proliferation and migration of glioma and is directly regulated by tumor-suppressive miR-124. FEBS Lett. 2013; 587:2455-60.

https://doi.org/10.1016/j.febslet.2013.06.018 PMID:23792158

19. Simon MP, Tournaire R, Pouyssegur J. The angiopoietin-2 gene of endothelial cells is upregulated in hypoxia by a HIF binding site located in its first intron and by the central factors GATA-2 and Ets-1. J Cell Physiol. 2008; 217:809-18.

https://doi.org/10.1002/jcp.21558

PMID:18720385

20. Weidner N. Current pathologic methods for measuring intratumoral microvessel density within breast carcinoma and other solid tumors. Breast Cancer Res Treat. 1995; 36:169-80.

https://doi.org/10.1007/BF00666038 PMID:8534865

21. Qu S, Hu T, Qiu O, Su Y, Gu J, Xia Z. Effect of Piezo1 Overexpression on Peritumoral Brain Edema in Glioblastomas. AJNR Am J Neuroradiol. 2020; 41:1423-29.

https://doi.org/10.3174/ajnr.A6638

PMID:32675337

22. Onishi M, Ichikawa T, Kurozumi K, Date I. Angiogenesis and invasion in glioma. Brain Tumor Pathol. 2011; 28:13-24.

https://doi.org/10.1007/s10014-010-0007-z

PMID:21221826

23. Osada H, Tokunaga $T$, Hatanaka H, Kawakami $T$, Tsuchida T, Abe $Y$, Tsugu A, Kijima H, Yamazaki $H$, Shima K, Osamura Y, Ueyama Y, Nakamura M. Gene expression of angiogenesis related factors in glioma. Int J Oncol. 2001; 18:305-309.

https://doi.org/10.3892/ijo.18.2.305

PMID:11172596
24. Nicolas S, Abdellatef S, Haddad MA, Fakhoury I, ElSibai M. Hypoxia and EGF Stimulation Regulate VEGF Expression in Human Glioblastoma Multiforme (GBM) Cells by Differential Regulation of the PI3K/RhoGTPase and MAPK Pathways. Cells. 2019; 8:1397. https://doi.org/10.3390/cells8111397 PMID:31698752

25. Solecki G, Osswald M, Weber D, Glock M, Ratliff $M$, Müller HJ, Krieter O, Kienast $\mathrm{Y}$, Wick W, Winkler F. Differential Effects of Ang-2/VEGF-A Inhibiting Antibodies in Combination with Radio- or Chemotherapy in Glioma. Cancers (Basel). 2019; 11:314.

https://doi.org/10.3390/cancers11030314

PMID: $\underline{30845704}$

26. Fidler IJ, Ellis LM. The implications of angiogenesis for the biology and therapy of cancer metastasis. Cell. 1994; 79:185-88. https://doi.org/10.1016/0092-8674(94)90187-2 PMID:7525076

27. Kloepper J, Riedemann L, Amoozgar Z, Seano G, Susek K, Yu V, Dalvie N, Amelung RL, Datta M, Song JW, Askoxylakis V, Taylor JW, Lu-Emerson C, et al. Ang2/VEGF bispecific antibody reprograms macrophages and resident microglia to anti-tumor phenotype and prolongs glioblastoma survival. Proc Natl Acad Sci U S A. 2016; 113:4476-81.

https://doi.org/10.1073/pnas.1525360113

PMID:27044098

28. Osawa T, Tosaka M, Nagaishi M, Yoshimoto Y. Factors affecting peritumoral brain edema in meningioma: special histological subtypes with prominently extensive edema. J Neurooncol. 2013; 111:49-57. https://doi.org/10.1007/s11060-012-0989-y PMID:23104516

29. Chinese Neurosurgical Society. [Consensus on the drug treatment of peritumoral brain edema]. Zhonghua Yi Xue Za Zhi. 2010; 90:5-9.

PMID:20356516 